\title{
Les " phrase " de Claude Simon : entre thème romanesque et réflexion linguistique
}

David Zemmour

\section{(2) OpenEdition}

1 Journals

Édition électronique

URL : http://journals.openedition.org/ccs/568

DOI : $10.4000 /$ ccs. 568

ISSN : 2558-782X

Éditeur :

Presses universitaires de Rennes, Association des lecteurs de Claude Simon

\section{Édition imprimée}

Date de publication : 30 avril 2007

Pagination : 43-59

ISBN : 9782354120122

ISSN : $1774-9425$

\section{Référence électronique}

David Zemmour, «Les «phrase » de Claude Simon : entre thème romanesque et réflexion linguistique », Cahiers Claude Simon [En ligne], 3 | 2007, mis en ligne le 20 septembre 2017, consulté le 03 mai 2019. URL : http://journals.openedition.org/ccs/568; DOI : 10.4000/ccs.568 


\section{Les « phrase» de Claude Simon : entre thème romanesque et réflexion linguistique}

David ZEMMOUR

«Phrase » est un mot que Claude Simon utilise peu dans son œuvre : 120 occurrences au total (76 au singulier, 44 au pluriel) du Tricheur au Tramway. C'est donc un mot qu'il ne prise guère. Si l'on ajoute à cela que l'écriture de Simon ne se conforme guère au modèle standard de la phrase telle qu'elle est définie par les grammaires traditionnelles, voilà qui devrait suffire pour considérer que la notion de phrase n'est pas pertinente chez Simon et qu'il vaut mieux s'intéresser à autre chose. Et pourtant.

Quoique réduit, le corpus n'en constitue pas moins une entrée fertile pour aborder la question de la réflexion métalinguistique chez Simon, une réflexion qui se développe naturellement dans les entretiens qu'il accorde, mais surtout au sein même de son œuvre. Que signifie le mot phrase en termes de dénotation et de connotation quand il est employé par Simon ? Y a-t-il construction d'un objet «phrase » à travers le discours simonien, et dans ce cas de quel objet s'agit-il ? Et surtout que peuvent nous apprendre les emplois du motphrase au sujet de la syntaxe simonienne ? Notre propos ici ne sera pas de tenter une description de la phrase simonienne et de son évolution, mais de déterminer dans quelle mesure Simon allie 
une conscience épilinguistique et une conscience métalinguistique de la phrase. En vertu de la première, Simon propose une réflexion spontanée et intuitive, comme pourrait le faire tout sujet parlant, à propos de la clarté, la logique ou la simplicité de la langue. En vertu de la seconde, il propose une réflexion sur la phrase en tant que notion grammaticale, dotée d'une définition précise dont on peut alors discuter la pertinence. Notre propos consistera à voir comment l'une et l'autre conscience se rejoignent, autrement dit comment la présence de la phrase dans des contextes et dans des acceptions peu grammaticales apriori peut cependant se lire comme le fruit d'une conscience proprement linguistique.

On commencera par un rapide aperçu des significations du mot en langue pour situer les emplois qu'en fait Simon par rapport à ce champ des possibles. Le Grand Robert de la Gangue Française (2001) divise l'article consacré à ce substantif en trois entrées d'importance inégale, en suivant une progression d'inspiration diachronique. La première entrée, assez brève, s'ouvre sur la définition donnée par Furetière : "Manière d'expression, tour ou construction d'un petit nombre de paroles ». Il s'agit là de l'acception courante du terme au $\mathrm{XVII}^{\mathrm{e}}$ siècle, à une époque où la phrase n'est pas encore entrée dans le champ de la grammaire. La phrase, c'est une expression, un syntagme, une formule, un tour, mais non pas une entité grammaticale, encore moins une mélodie. C'est en vertu de cette acception que faire desphrases signifie " arranger, disposer les mots de la langue en vue de produire un effet agréable », et dans un contexte plus péjoratif, « avoir recours à des façons de parler prétentieuses ».

La seconde entrée de l'article "phrase », de loin la plus fournie, concerne l'acception grammaticale du terme. Elle se divise en deux parties. La première est strictement linguistique et suit une progression chronologique, de Du Marsais, l'un des principaux théoriciens de la phrase au XVIII ${ }^{\mathrm{e}}$ siècle, à Emile Benveniste, plus près de nous, pour cerner les différents critères définitoires possibles, nombreux et parfois divergents. De fait, la notion de phrase s'est grammatisée au XVIII ${ }^{\circ}$ siècle, subissant au fil de cette période des sédimentations définitoires aboutissant à une sorte d'agrégat de critères hétérogènes figés artificiellement après la Révolution à des fins utilitaires, en 
particulier dans le but d'enseigner la langue écrite aux écoliers. C'est pourtant sur ce socle épistémologique peu stable que s'est développée aux $\mathrm{XIX}^{\mathrm{e}}$ et $\mathrm{XX}^{\mathrm{e}}$ siècles la réflexion linguistique traitant de la phrase. La seconde partie de cette entrée s'attache, elle, à appréhender la phrase en tant que fait de style (par exemple « une phrase nerveuse ») et d'oralité (" échanger quelques phrases»).

La troisième et dernière entrée, très courte, s'intéresse à la phrase musicale, une acception dont la première attestation est datée de 1742 (ce qui ne veut naturellement pas dire que le mot ait été employé pour la première fois dans ce sens cette année-là), et l'on ne manque pas de constater que cette évolution sémantique coöncide avec celle de la phrase grammaticale.

Ce rapide tour d'horizon des champs sémantiques de la phrase étant accompli, il devient possible de situer les emplois du mot par Simon avant de les caractériser.

On distinguera ces emplois, selon qu'ils ont lieu au sein de l'œuvre littéraire ou bien lors d'entretiens. C'est bien le premier cas qui nous intéresse, mais l'éclairage de l'un par l'autre peut être fécond. En effet, dans les entretiens qu'il accorde, Simon parle de phrase. Reste à savoir qui de l'intervieweur ou de l'interviewé suscite l'emploi du terme. Les enjeux ne sont certainement pas les mêmes selon que le mot est prononcé ou même simplement suscité par l'un ou l'autre. L'examen d'un certain nombre d'entretiens laisse apparaître une fréquence d'emplois relativement faible, mais surtout, les «phrase» surviennent sans avoir été directement suscités par la question. C'est donc spontanément que Simon est amené à aborder le sujet. Quant aux sens qu'il lui donne, deux orientations se dégagent. La première fait de phrase un équivalent de mot,formule, propos, employé lorsque Simon veut introduite une citation (par exemple «Dans les dernières pages du roman, la phrase: "Mais comment était-ce, comment savoir ?" revient comme un leitmotiv $\left.»^{1}\right)$. Mais on remarque aussi de la part de Simon l'emploi de phrase dans une acception explicitement grammaticale :

1 Claude Simon, "L'inlassable réancrage du vécu », Entretien avec Mireille CalleGruber (1993), in Mireille Calle-Gruber, Te Grand Temps. Tissai sur l'oeuvre de Claude Simon, Lille, Presses Universitaires du Septentrion, 2004, p. 237 
Tout bouge, rien n'est fixe. Le langage lui aussi est naturellement mouvant. On ne peut pas s'exprimer en 1960 avec la phrase de Stendhal, ce serait se promener en calèche!!

Je n'essaye pas de rendre, de décrire des pensées, mais de rendre des sensations. Or, la phrase (dans son organisation) convient fort mal à ce que je veux faire. ${ }^{2}$

J'étais hanté par deux choses : la discontinuité, l'aspect fragmentaire des émotions que l'on éprouve et qui ne sont jamais reliées les unes aux autres, et en même temps leur contiguïté dans la conscience. Ma phrase cherche à traduire cette contiguïté. $^{3}$

Ces trois déclarations sont contemporaines les unes des autres, tenues à l'automne 1960 lors de la publication de Fa Roule des Flandres. Dans le premier extrait, Simon parle avec humour de la phrase alors que la question posée portait sur sa participation au Manifeste des 121. La digression de son propos se fonde sur la nécessité, y compris pour la langue, de suivre les mouvements de l'histoire. Dans les deux déclarations suivantes, la question n'appelle pas davantage la notion de phrase. Dans la troisième en particulier, le mot survient au cours d'une réponse longue à une question brève et ouverte qui concerne le thème principal de Fa Route des Flandres. Dans ces deux derniers exemples, l'emploi des déterminants est éclairant : il permet d'opposer « la » phrase, celle des grammaires traditionnelles, et «ma » phrase, celle de Simon, une phrase dont il annonce qu'elle se plie à d'autres règles. On voit bien le lien que tisse Simon entre syntaxe et perception : la syntaxe doit se mettre pour lui au service de la perception, ou plus exactement de la succession des différentes perceptions dans la conscience, mais cela passe par une syntaxe alternative à celle de la phrase, qui est absolument inapte à restituer la logique de la sensation et de l'émotion.

Le constat qui s'impose ici est que lorsque Simon parle de phrase

Madeleine Chapsal, « Entretien avec Claude Simon », L'Express, 10 nov. 1960.

2 Hubert Juin, «Les secrets d'un romancier », Les Lettres Françaises, 6-12 octobre 1960. Nous soulignons.

3 Claude Sarraute, "Avec La Route des Flandres, Claude Simon affirme sa manière ", Le Monde, 8 oct. 1960. Nous soulignons. 
lors d'entretiens, il s'agit le plus souvent de l'entité syntaxique, alors même que la question posée ne convoquait pas apriori une telle acception (il n'y est d'ailleurs pas question de phrase du tout). Le terme est employé à la faveur de digressions indépendantes des questions et donc parfaitement libres, attestant que la phrase en tant que fait de syntaxe est une préoccupation consciente de Simon, même si, il faut bien le dire, le contexte critique plus général l'y conduit en raison des commentaires acerbes dont son style a pu faire l'objet à l'époque.

$A$ contrario, lorsqu'il aborde les questions pour lui essentielles du style et en particulier du rythme, Simon recourt à une autre terminologie. Ainsi, à Madeleine Chapsal qui l'interroge sur l'absence de ponctuation dans son écriture, Simon parle volontiers de tempo ${ }^{1}$. Plus récemment, à Philippe Sollers qui l'interroge à propos de Céline, il déclare : «Pourquoi est-ce si extraordinaire ? Parce que c'est très bien écrit. Parce qu'il y a une musique, parce qu'il y a une cadence. Voilà ! c'est tout $»^{2}$. L'évocation du rythme et du phrasé de l'écriture appelle, on le voit, une terminologie musicale qui n'inclut pas le terme de phrase que Simon semble réserver à une réflexion plus directement syntaxique, quand bien même celle-ci induit des phénomènes rythmiques. Les phrase de Simon dans le cadre des entretiens qu'il accorde témoignent donc d'une réflexion linguistique permanente, concernant en particulier la nécessaire adéquation de la syntaxe à l'écriture de la sensation.

De semblables discours apparaissent-ils au sein de l'œuvre romanesque ? De fait, de tels emplois de phrase sont rares, Simon se livrant fort peu à des considérations explicites sur la phrase en tant qu'entité grammaticale. Cas isolé, cet extrait du Vent expose les commentaires pessimistes d'Antoine Montès, personnage principal du roman, sur les limites de la phrase :

Car, me dit-il, ce fut ainsi que cela se passa, en tout cas ce fut cela qu'il vécut, lui : cette incohérence, cette juxtaposition

2 Philippe Sollers, «La sensation, c'est primordial », Le Monde, 19 sept. 1997. 
brutale, apparemment absurde, de sensations, de visages, de paroles, d'actes. Comme un récit, des phrases dont la syntaxe, l'agencement ordonné - substantif, verbe, complément - seraient absents. Comme ce que devient n'importe quel article de journal (le terne, monotone et grisâtre alignement de menus caractères à quoi se réduit, aboutit toute l'agitation du monde) lorsque le regard tombe par hasard sur la feuille déchirée qui a servi à envelopper la botte de poireaux et qu'alors, par la magie de quelques lignes tronquées, incomplètes, la vie reprend sa superbe et altière indépendance, redevient ce foisonnement désordonné, sans commencement ni fin, ni ordre, les mots éclatants d'être de nouveaux séparés, libérés de la syntaxe, de cette fade ordonnance, ce ciment bouche-trou indifféremment apte à tous usages et que le rédacteur de service verse comme une sauce, une gluante béchamel pour relier, coller tant bien que mal ensemble, de façon à les rendre comestibles, les fragments éphémères et disparates de quelque chose d'aussi indigeste qu'une cartouche de dynamite ou une poignée de verre pilé : grâce à quoi (au grammairien, au rédacteur de service et à la philosophie rationaliste) chacun de nous peut avaler tous les matins, en même temps que les tartines de son petit déjeuner, sa lénifiante ration de meurtres, de violences et de folie ordonnés de cause à effet $[\ldots] .(V, 137)$

Un tel exemple est remarquable pour au moins deux raisons. D'abord phrase a son sens grammatical en quelques autres occurren$\operatorname{ces}^{1}$, mais celle-ci constitue la seule qui soit directement liée à une réflexion linguistique ; ensuite cette réflexion fait l'objet d'un long développement exprimant ce que Simon dira plus tard dans le cadre d'entretiens, à savoir l'inaptitude ontologique de la phrase à restituer une expérience sensorielle en raison des principes d'organisation logique, rectionnelle et rationnelle qui la constituent. Pour cette raison, la phrase est, si l'on en croit l'extrait cité, l'outil de travail du grammairien, du journaliste et du philosophe rationaliste, parce que

Par exemple : " "Tu tiens à le savoir espèces de putains ?", le premier membre de la phrase au singulier, le second au pluriel [...]» $(S P, 231)$; ou encore : " [la voix] courant pour ainsi dire sur sa lancée, mettant bout à bout mots et phrases suivant une syntaxe, un ordre machinal et d'ailleurs sans importance - l'important étant la non-cessation du son, du bruit $[\ldots] »(V, 118)$, etc. 
par son essence, elle se fait l'expression du compte rendu ou du raisonnement, mais elle n'est assurément pas l'outil du romancier.

Ce sont là les lignes les plus explicites que Simon ait écrites au sujet de la phrase grammaticale dans ses romans. Observons qu'elles sont tirées du Vent, premier temps d'une évolution décisive dans la pratique romanesque de Simon qui se poursuivra dans L'Herbe et s'accomplira dans La Route des Flandres. Observons encore que si ces réflexions sont formulées par Montés, tout porte à penser que Simon les fait siennes au sein de ce qui ressemble fort à un art poétique.

Un tel exemple, on l'a dit, reste exceptionnel. Dans la majorité de ses emplois, phrase désigne un discours, des paroles prononcées ou encore des mots écrits. Caractéristique la plus immédiatement perceptible, ces emplois sont associés à l'idée d'incomplétude. Dans nombre d'occurrences, phrase dénote un fragment de discours, qu'il s'agisse d'une émission ou d'une perception incomplète. Les personnages ne finissent pas leur phrase, ou bien ils ne l'entendent pas entièrement, que ce soit par inattention ou en raison de bruits parasites :

[...] disparus avant même d'avoir fini le mouvement, la phrase $[\ldots](\boldsymbol{V} 155)$

[...] comme si dans un salon quelqu'un l'avait [...] interrompu au milieu d'une phrase $[\ldots](R F, 203)$

Il commence une phrase mais il est interrompu [...] $(T ; 808)$

Les exemples sont très nombreux où la phrase apparaît ainsi réduite à une succession de fragments ou à des discours incomplets. Ce faisant, Simon va à l'encontre d'un procédé narratif stéréotypé par lequel l'interruption a pour fonction d'attirer l'attention sur l'événement nouveau qui survient et impose la cessation de tout ce qui pouvait avoir lieu auparavant : « il n'eut pas le temps de finir sa phrase que... » ou "à peine avait-il commencé sa phrase que... ». Rien de tel ici puisque c'est l'interruption elle-même - et non ce qui la cause - qui fait événement. Les emplois de phrase s'inscrivent dans 
le cadre d'une relation duale, parfois entre parole et silence, parfois entre plusieurs paroles. Ils marquent d'un côté la hantise d'un discours qui s'épuise, et de l'autre une progression de la parole sur le mode du chevauchement, ce qui revient dans les deux cas à donner de la parole une représentation en creux : la parole, c'est d'une part lorsqu'il n'y a pas silence, d'autre part quand autrui ne parle pas. La représentation des discours dans les romans de Simon a ceci de particulier qu'elle fait de la parole une fin en soi. Nombreux sont les personnages qui parlent avant tout pour parler, indépendamment de ce qu'ils ont à dire, attestant par là même leur existence et leur présence. Dans La Route des Flandres, les occupants du wagon de prisonniers ou encore Georges dans la chambre d'hôtel en compagnie de Corinne ne recherchent pas autre chose, «l'important étant la non-cessation du bruit » pour reprendre une formule du Vent déjà mentionnée.

La parole peut paraître vaine ou dérisoire au vu des circonstances dans lesquelles elle est parfois proférée. Il n'en demeure pas moins que sa présence atteste un enjeu et les emplois de phrase attestent cette réflexivité du discours simonien qui s'interroge sur sa portée, sa fonction, ou encore les interactions personnelles que tout discours implique. Ajoutons que la narration est toujours focalisée chez Simon. Les événements relatés sont toujours appréhendés à travers le prisme d'une focalisation, et si la phrase est représentée comme étant morcelée, c'est d'abord parce qu'elle est perçue comme telle. Comment ne pas retrouver là l'expression d'une conviction toute simonienne, à savoir que la connaissance que nous avons du monde est inévitablement partielle, ne pouvant se faire que par bribes ? À cet égard, l'emploi du mot phrase est à situer au cœur de la problématique simonienne de la perception : en tant qu'unité de discours, elle relève à la fois de l'émission et de la réception; elle est à la jonction de l'événement et de sa perception par les sens.

Et pas seulement par l'ouïe, d'ailleurs. Elle convoque d'autres modes d'appréhension du réel. Pour s'en rendre compte, on peut tenter une classification des emplois. On peut ainsi distinguer phrase émise et phrase perçue, et pour ces deux catégories, distinguer les cas où la phrase est orale de ceux où elle est écrite. Soient en somme 
quatre catégories, selon que la phrase est proférée, écrite, entendue ou lue. De fait, il semble qu'il y ait une spécialisation des emplois, la perception concernant plutôt la phrase écrite, l'émission la phrase orale. Les phrases chez Simon sont donc lues plutôt qu'entendues, et prononcées plutôt qu'en train d'être écrites. Cela étant posé, ces catégories ne sont pas nécessairement étanches. On observe ainsi une perméabilité entre l'oral et l'écrit d'une part, entre émission et perception d'autre part. Dans Les Géorgiques, il arrive à L.S.M. de dicter à son secrétaire des phrases qui sont donc dites avant d'être écrites. L'Acacia fournit un autre exemple, lorsqu'il est question d'un faire-part de décès que la mère envisage de faire imprimer, "un rectangle de carton discrètement bordé de noir, orné d'une mince croix noire au-dessus du nom, de la date ([...]), des courts versets d'Ozanan ou de Job et de la phrase (le cri) qui peut-être lui vint à la gorge, l'étouffant, la suffocant» ÇA, 278). La phrase est envisagée comme phénomène sonore (expression de la douleur de l'épouse devenue veuve) puis écrit (son impression sur le faire-part). Elle apparaît dans un contexte où se heurtent deux formes d'expression celle de la douleur officielle, conventionnelle, presque mondaine au moyen de formules types, et l'autre, moins articulée et plus intime. Phrase criée puis écrite, phrase dictée, phrase lue pour être hurlée, elle apparaît comme un point de jonction entre l'oral et l'écrit ainsi qu'entre l'émission et la perception. Dans tous les exemples de ce type, phrase cristallise une idée d'incompatibilité entre les supports ou entre les partis qu'elle implique. Le passage de l'oral à l'écrit correspond à un changement de support, et ce changement est associé à une altération (consentie ou non) et parfois même une annulation du message. Pas de reproduction à l'identique. Une phrase répétée ou reproduite, ce n'est déjà plus la même phrase. De l'une à l'autre, il y a nécessairement altération.

L'autre porosité concerne émission et réception de la phrase, qui dans ces situations qui relèvent à la fois d'un dire et d'un entendre, problématisent cette relation dans la mesure où elle n'est jamais correctement ou complètement perçue. C'est là encore un phénomène d'altération dans la transmission. La phrase est cet objet de roman intermédiaire, avons-nous dit, qui se situe entre le perceptible et le 
perçu. Elle pose le problème de l'adéquation entre ce qui est et ce qu'on perçoit. Le questionnement sur ce clivage est constant chez Simon, et plus particulièrement dans $\mathrm{Fa}$ Route des Flandres où revient comme un leitmotiv l'interrogation «Comment savoir? ». Mais lorsqu'il s'agit de phrase, ce questionnement s'appuie sur un cas d'école, puisqu'il concerne des fragments de discours. Si pour bien des raisons (absence de témoin, défaut de mémoire), il est impossible de savoir " comment c'était », il paraît en revanche envisageable de restituer des paroles telles qu'elles ont été dites et afortiori telles qu'elles ont été écrites puisque leur statut de discours imprimé leur garantit en théorie un minimum de pérennité. Tel n'est pourtant pas le cas. Comme le montrent les emplois du mot phrase, Simon étend la systématisation du doute jusqu'au discours. Répéter ou reproduire, c'est déjà dire autre chose, et cela ne permet assurément pas de savoir ce qui s'est initialement dit. L'usage du magnétophone, tourné en dérision dans LeJardin des Plantes, illustre bien cette méfiance de Simon envers tout ce qui a la prétention de capter et fixer l'événement. C'est ainsi que toute phrase apparaît comme déjà de seconde main. Transmissible par nature, elle est vouée à l'altération.

Cette propension à la détérioration est liée à un changement de support qui charge le discours d'une matérialité : phrase imprimée dans un journal, ou bien écrite dans un cahier, ou encore gravée sur une stèle funéraire, etc. C'est de fait une qualité que Simon confère à sesphrase, en évoquant régulièrement le support concret au moyen duquel elles s'expriment. En témoigne, dans Les Corps conducteurs, cette phrase inscrite sur un oriflamme balancé par le vent : "Au gré de celui-ci apparaissent et disparaissent les mots ou des portions de mots formant la phrase O CRUX AVE SPES UNICA brodée en fils d'or au-dessous d'une croix entourée de rayons. " (CC, 41). Simon rend tangible, si l'on peut dire, le fil de la phrase, devenue objet concret par la broderie et l'oriflamme.

Par son instabilité statutaire (orale ou écrite, émise ou perçue), la phrase engage donc la présence de son support matériel faisant corps avec elle, au point de compromettre son intégrité lorsqu'il est lui-même défaillant ou abîmé. Et lorsque la phrase n'est pas directement associée à son support matériel, il n'est pas rare qu'elle re- 
trouve une concrétude par le jeu de la métaphore. C'est ainsi qu'elle apparaît sous la forme de « fragments », « bouts », « bribes », " lambeaux », « membres » voire sous forme de « matière gluante ${ }^{1}$. C'est bien alors la métamorphose qui donne consistance à la phrase pour en faire un objet soumis à la description parmi les autres. Et de même que les autres objets de l'univers simonien, elle est exposée aux effets du temps, c'est-à-dire à l'érosion et à la dislocation. Chez Simon, la matérialité ne va pas sans la fragmentation. Au contraire d'une norme fixe et durable, elle se caractérise par sa fragilité, sa précarité et jusqu'à sa déliquescence. Cette matérialité fragmentaire de la phrase est parfois même dépassée pour accéder à un statut organique aux frontières de la personnification :

[...] ils se mirent à parler entre eux, échangeant des embryons de phrases sur un ton geignard et sentencieux [...]. $(C R, 133)$

[...] tandis qu'il écoutait se succéder les phrases, ou plutôt les embryons de phrases, le plus souvent laissées en plan avant la fin, et quelquefois en plein milieu, et quelquefois tout juste commencées comme si celui qui les prononçait essayait des pistes successives aussitôt abandonnées pour une qui lui paraissait meilleure [...]. $(V, 53)$

[...] disant : ... « une puante momie enveloppée et étranglée par le cordon ombilical de kilomètres de phrases enthousiastes tapées sur ruban à machine par l'enthousiaste armée des correspondants étrangers de la presse libérale. » $(P, 420)$

Associée à « embryon » ou « cordon ombilical », la phrase est du domaine du vivant. Ou plus exactement le recours à de telles métaphores permet d'établir une analogie entre la phrase et l'embryon, analogie par laquelle la phrase est au discours ce que l'embryon est à l'homme ou à la vie, c'est-à-dire incomplète et virtuelle. Le contexte dans lequel apparaissent ces expressions est connoté très négative-

1 La phrase est présente à l'état de «fragments » dans $S P, 119$; réduite à des «bouts » dans Gul., 270 ; par « bribes » dans Gul., 101, RF, 352, Hist., 26, T, 775 ; en «lambeaux» dans $V, 119$, Hist, 24 ; par «membres» dans $C C, 52$; et sous forme de « matière gluante» dans $V, 58$. 
ment, et ne laisse aucun doute sur la signification que Simon leur donne : il ne s'agit pas de la promesse d'un accomplissement ou d'une naissance à venir. Dans un cas « les phrases, ou plutôt les embryons de phrases" sont «laissées en plan avant la fin», et dans l'autre «le cordon ombilical de kilomètres de phrases » enveloppe une "puante momie». La phrase est sans équivoque associée à la mort. Chargée d'une force vitale, elle ne parvient pas à terme. Avorton langagier, discours mort-né, la phrase n'est pas viable ${ }^{1}$.

Incomplète et altérée dans son signifiant, la phrase est réduite à un magma sonore et vide de toute signification. Cette détérioration aboutit à l'écrit à des textes illisibles, par exemple une épitaphe à peine déchiffrable dans Les Géorgiques (G, 162-165). De même, à l'oral, les phrases sont réduites à des bruits :

[...] les paroles ne lui parvenant même pas, ou du moins ne parvenant même pas à se transformer (les mots, les signes verbaux et sonores) en quelque phrase qui signifiât pour elle autre chose qu'un bruit, un son [...] (Herbe, 250)

[...] ce qui n'était que sons [...] devenant de nouveau des mots puis des phrases peut-être simplement alignés bout à bout dans l'unique raison de faire du bruit [...] (Hist., 182)

À cette altération du signifiant correspond inévitablement celle du signifié. On aboutit fréquemment à son anéantissement comme l'attestent les adjectifs ou groupes adjectivaux qui caractérisent la phrase : "privés de signification", "vidées de sens", "vides de sens », « insignifiante », ou « incompréhensible $»^{2}$.

1 Le thème de l'embryon reparaît au terme d'Histoire pour représenter avec humour le narrateur : «[...] la femme penchant son mystérieux buste de chair blanche enveloppé de dentelles ce sein qui déjà peut-être me portait dans son ténébreux tabernacle sorte de têtard gélatineux lové sur lui-même avec ses deux énormes yeux sur sa tête de ver à soie sa bouche sans dents son front cartilagineux d'insecte, moi ?...» (Hist., 402). C'est là un paradoxe du roman qui s'achève par l'image du narrateur (peut-être du romancier) en gestation, au moyen d'une phrase qui, sans être véritablement inachevée, reste ouverte à de possibles prolongements. Le contexte, quoique chargé d'autodérision, est beaucoup moins négatif que précédemment.

2 Respectivement dans Gul., 73, SP, 131, I, 91, $R F$, 353, et pour « incompréhen- 
Il semble qu'on en revienne ici à une réflexion plus directement linguistique. La représentation dysphorique de la phrase en tant que thème romanesque rejoint les réflexions plus directement métalinguistiques de Simon sur la notion en tant qu'unité non pertinente pour l'écriture de la sensation et de l'émotion. L'un et l'autre se rejoignent également par le jeu de la spécularité. Phrase apparaît en effet dans des contextes où un personnage est en train d'écrire, et ces mises en scène de l'acte d'écriture sont l'occasion de faire émerger une représentation particulière de la phrase. Ce type de situation n'est pas toujours associé à l'emploi du mot phrase, comme le montrent les pages représentant à sa table de travail le scripteur de La Bataille de Pharsale, mais lorsqu'il l'est, on est frappé de constater à quel point le texte (dans la fiction) auquel cette activité d'écriture donne lieu est dévalorisé

[...] pensant (Georges) : «Alors il peut sans doute recommencer à y croire, à les aligner, les ordonner élégamment les uns après les autres, insignifiants, sonores et creux, dans d'élégantes phrases insignifiantes, sonores, bienséantes et infiniment rassurantes, aussi polies, aussi glacées et aussi peu solides que la surface miroitante de l'eau recouvrant, cachant pudiquement...» $(R F, 353)$

On dirait que les mots assemblés, les phrases, les traces laissées sur le papier par les mouvements de troupes, les combats, les intrigues, les discours, s'écaillent, s'effritent et tombent en poussière $[\ldots] .(\mathrm{G}, 76)$

L'exemple tiré du Palace mentionné plus haut va dans le même sens. Insignifiance, putréfaction, érosion : tel est le sort dévolu aux phrases écrites. Elles apparaissent comme inutiles, vaines et précaires. Et à chaque fois, associée à ces vaines et stupides tentatives d'écriture, il y a la guerre qui a fait son œuvre et qui oblige à interroger le rapport des mots aux choses. Il semblerait que la guerre soit un contexte dans lequel «phrase » ne signifie rien. Dans une œuvre qui fait de la guerre un thème de prédilection, la phrase en tant que modèle de production langagière ne s'avère plus pertinente. 
Il apparaît également que la phrase en tant que norme écrite dévalorisée est régulièrement associée à une figure d'autorité, elle aussi fortement contestée. Qui sont les auteurs des phrases mentionnées ci-dessus ? Respectivement le père de Georges dans La Route des Flandres, les «correspondants étrangers de la presse libérale » pendant la guerre d'Espagne dans Le Palace, et L.S.M., l'ancêtre Conventionnel dans Les Géorgiques. Les rapports de dépendance hiérarchique, idéologique ou généalogique mis en place par ces figures d'autorité sont à l'image de ceux qui peuvent exister au sein de la phrase : contestés. L'emploi du motphrase chez Simon s'inscrit donc dans un contexte de contestation des rapports de dépendance, hiérarchiques ou syntaxiques. Phrase apparaît d'ailleurs pour un nombre non négligeable d'occurrences dans un contexte politique fortement marqué :

Et sur l'affiche le dessin représentant le type est barré par une croix péremptoire et il y a une phrase en lettres énormes qui veut dire à peu près «Plus de ça! » ou «C'est fini maintenant. Le gouvernement donne du travail à tous!» (Gul., 316)

[...] les proclamations de grèves, les annonces de meetings, les infatigables phrases de colère, de révolte, d'infatigable espoir [...]. $(\mathrm{V}, 190)$

[...] la phrase maintenant connue par cœur se reformant d'elle-même dans l'esprit [...] :

\section{¿ QUIEN HA MUERTO A SANTIAGO ?}

(Et voici la phrase que je propose d'ajouter immédiatement à la suite pour compléter le paragraphe quatre) $\{C C, 214)$

[...] les voix des interprètes finissant de traduire sa dernière phrase $[\ldots] .(I, 53)$

Affiches de propagande, titres et articles de journaux qui rendent compte de la guerre d'Espagne, congrès littéraire où règne une hégémonie idéologique qui interdit tout débat, accueil de sommités mondiales par des dignitaires soviétiques en quête de respectabilité internationale : qu'elle soit du côté des opprimés ou des oppres- 
seurs, la phrase chez Simon est souvent politique, historique et idéologique, associée à un contexte militant ou totalitaire. C'est simultanément un discours normé, un attirail de conventions et de lois et un outil de propagande. Simon se tient à distance de ce type de discours par une tonalité ironique, par le jeu des guillemets ou plus généralement par celui des discours rapportés. La dernière citation est à cet égard exemplaire. C'est en effet dans L'Invitation que la fréquence de " phrase(s) » est la plus élevée : sept occurrences pour 87 pages. C'est également l'œuvre de Simon où le contexte politique et idéologique est le plus prégnant et le plus pesant, en particulier pour le personnage principal qui marque ses distances en refusant de signer une pétition. Les " phrase(s) » de cette œuvre sont soit des discours d'officiels soviétiques, soit des conversations mondaines entre des invités qui ne se connaissent pas, c'est-à-dire soit des discours taillés dans une langue de bois, soit des discours convenus qui sont à eux-mêmes leur propre fin. Faut-il préciser que L'Invitation est le seul récit de Simon qui n'ait pas droit à la mention « roman » ? Fautil préciser encore que L'Invitation est écrit au moyen d'une syntaxe relativement conventionnelle ? En somme, plus il y a de "phrase» et de phrases, et moins il y a de roman. Parce qu'elle repose sur des fondements hiérarchiques et qu'elle est d'essence idéologique, la phrase n'est manifestement pas du côté de l'art et de la création littéraire, pas plus qu'elle n'est, selon les termes de Georges Molinié, « du côté de la liberté ou de la vie ».

Faut-il aller jusqu'à dire que les discours de Simon sur la phrase sont d'essence politique et idéologique ? De fait, il ne sont pas sans rappeler le sentiment d'attraction-répulsion de Roland Barthes face à la structure phrastique et la grammaire, notions toujours connotées négativement chez lui dans la mesure où elles sont rattachées à la naissance du dogme grammatical au XVII ${ }^{\mathrm{e}}$ siècle et sont considérées comme le produit d'un asservissement de type politique ${ }^{2}$ :

1 Georges Molinié, «À propos de la notion de phrase », Mélanges Lanher-Lorraine vivante: hommage à Jean lumber, Roger Marchai et Bernard Guidot eds. (Nancy, Presses Universitaires de Nancy, 1993), p. 393.

2 Voir sur ce point l'analyse de Gilles Philippe dans Sujet, verbe, complément, Le moment grammatical de la littératurefrançaise, 1840-1940 (Paris, Gallimard, « Bibliothèque des idées », 2002), p. 203. 
[O]n peut se demander si la phrase, comme structure syntaxique pratiquement close, n'est pas elle-même, déjà, une arme, un opérateur d'intimidation [...]. [D]ans la vie courante, dans la vie apparemment libre, nous ne parlons pas par phrases. Et, inversement, il y a une maîtrise de la phrase qui est très proche d'un pouvoir : être fort, c'est d'abord finir ses phrases. La grammaire elle-même ne décrit-elle pas la phrase en termes de pouvoir, de hiérarchie: sujet, subordonnée, complément, rection, etc. ?

Cette position peut à certains égards faire penser au rapport qu'entretient Simon avec la phrase. En effet, dans un cas comme dans l'autre, il y a ce constat initial que la phrase incarne une organisation hiérarchique, on l'a vu plus haut avec les déclarations de Simon lui-même. Mais le rapprochement s'arrête là, car les deux discours divergent: Barthes en vient à une réflexion politique et idéologique, faisant de la phrase un instrument de pouvoir. Rien de tel chez Simon qui se borne à constater l'incapacité de la phrase à retranscrire l'architecture sensorielle, dont les principes d'organisation et d'articulation sont incompatibles avec ceux de la syntaxe phrastique. Au demeurant, cela n'empêche pas Simon de considérer comme contestataire sa pratique de l'écriture, ainsi qu'il le déclare à André Bourin qui le questionne sur son engagement et sa participation au Manifeste des 121: « ce que je fais en tant que romancier et en tant que citoyen, c'est, sur deux plans différents mais parallèles, la même chose : appelons cela, si vous voulez, la contestation. Contestation, d'une part, de formes établies et, me semble-t-il, périmées, mortes ; d'autre part, de structures sociales établies et, me semble-t-il encore, périmées elles aussi $»^{2}$. Redisons avec Simon que ces deux terrains sont dissociés. Le travail du romancier, et en particulier le rapport à la phrase, ne relève pas d'un engagement politique qui serait fondé sur le constat d'un fascisme de la phrase. Il n'en demeure pas moins que l'écriture simonienne est un écriture contre, s'opposant d'ellemême à des formats stéréotypés et historiquement datés, mais sans les exclure. Au contraire, elle les intègre par incorporation pour les

Roland Barthes, extrait d'une conférence de 1973 publiée dans les Euvres complètes (Paris, Seuil, 1993-5), t. II, p. 1612.

${ }^{2}$ André Bourin, « Techniciens du roman », Les Nouvelles littéraires, 22 déc. 1960. 
donner en représentadon et en pointer les insuffisances.

Ainsi Simon ne disserte-t-il guère sur la phrase en tant qu'entité linguistique. Son utilisation ne coïncide pas avec une réflexion directement syntaxique mais avec une mise en représentation marquée par une indéniable constance d'un roman à l'autre, par-delà les manières successives de Simon. Et par cette mise en représentation, Simon tient sur la phrase un discours que l'on peut résumer ainsi : à la fois entité linguistique et objet sensible, la phrase concentre les enjeux d'une réflexion sur l'écriture de la sensation fondée sur les principes d'absence d'organisation hiérarchique et de fragmentation. Aux modèles normatifs et archi-codifiés, Simon oppose une phrase fragile, en proie au travail destructeur du temps, à l'existence précaire, inachevée, qui refuse tout modèle, et qu'il faut à chaque fois reconstruire, reconcevoir et, sans doute, si l'on veut s'essayer à une description de la syntaxe simonienne, rebaptiser. 